\title{
Spatially resolved spectra in semiconductor quantum structures: Spatially averaged spectra compared to far-field spectra
}

\author{
G. Pistone, S. Savasta, O. Di Stefano, and R. Girlanda \\ INFM and Dipartimento di Fisica della Materia e Tecnologie Fisiche Avanzate, Università di Messina, Salita Sperone 31 , I-98166 \\ Messina, Italy
}

\begin{abstract}
The optical spectra of homogeneous surface systems can display remarkable differences in the near and far zones. The spectral changes occur due to the loss of evanescent modes in the far zone. These changes clearly show that near-field optical spectroscopy and microscopy, besides resolving nanometric structures give also access to excitations that cannot be revealed in the far zone. Are these spectral changes detectable in real systems affected by disorder and imperfections? We address this issue by presenting a theoretical analysis of the local optical properties of semiconductor quantum wells including the effects of interface fluctuations. In particular we compare the far-field absorption spectrum with spatially averaged absorption spectra calculated at different spatial resolutions. We find that summing up local optical spectra does not reproduce the global spectrum in contrast to findings at diffraction-limited resolutions.
\end{abstract}

DOI: $10.1103 /$

\section{INTRODUCTION}

Near-field microscopy and spectroscopy extend the possibility of optical characterization of surface systems to nanometer dimensions. Recent measurements based on spatially-resolved photoluminescence provided direct information on the spatial and energy distribution of light emitting nanometric centers ${ }^{1-5}$ of semiconductor quantum structures. Moreover near-field spectroscopy offers unique attributes in addition to high spatial resolution which might be explored in future experiments. For example succeeding in confining the optical excitation to a very small volume below the diffraction limit, implies the presence of optical fields with high lateral spatial frequencies able to excite surface states with high $k$ vectors not accessible by far-field optical excitations. As a consequence optical spectra of homogeneous surface systems can display remarkable differences in the near and far zones. ${ }^{6,7}$ The spectral changes occur due to the loss of evanescent modes (corresponding to modes with high lateral spatial frequencies) in the far zone. These changes have been analyzed theoretically in ideal disorderfree surface systems where in-plane $k$ vectors are good quantum numbers.

Here we analyze these additional opportunities of nearfield spectroscopy in a realistic surface system affected by disorder effects. Inhomogeneities and disorder effects result in surface quantum states, eventually localized, with mixed in-plane $k$ vectors (the in-plane $k$ vector is no more a good quantum number). To what extent these spectral changes are detectable in real systems affected by disorder and imperfections? Does near-field microscopy provide just a spatial selection of inhomogeneous surface systems ${ }^{8,9}$ or, in addition, does it enable the optical detection of states not accessible by far-field optical probes? These addressed questions are directly related to the following question: are spatially averaged near-field spectra equal to far-field spectra? In order to isolate spectral changes due to excitation of states not accessible by far-field optical probes, we compare far-field absorp- tion spectra with spatially averaged absorption spectra. These averaged spectra are composed summing up local spectra obtained centering the confined illuminating beams on a fine mesh of points on the sample surface. In particular we study the local optical absorption of semiconductor quantum wells (QW's) including the effects of interface fluctuations. Interface fluctuations in QW's result in an effective twodimensional (2D) spatially correlated random potential that tends to localize the center of mass (c.m.) motion of excitons $^{8,10}$ and produces an inhomogeneous Gaussian-like absorption line. ${ }^{11,12}$

\section{THEORY}

From classical electromagnetism, the total absorbed power under local illumination is proportional to ${ }^{8,9,13}$

$$
\alpha(\omega)=\operatorname{Im} \int \mathbf{P}^{*}(\mathbf{r}, \omega) \mathbf{E}(\mathbf{r}, \omega) d \mathbf{r},
$$

where $\mathbf{P}(\mathbf{r}, \omega)$ is the polarization density of the sample, induced by the electric field $\mathbf{E}(\mathbf{r}, \omega)$. According to the general linear response theory, the macroscopic polarization $\mathbf{P}(\mathbf{r}, \omega)$ can be written as

$$
\mathbf{P}(\mathbf{r}, \omega)=\int \overleftrightarrow{\chi}\left(\mathbf{r}, \mathbf{r}^{\prime}, \omega\right) \mathbf{E}\left(\mathbf{r}^{\prime}, \omega\right) d \mathbf{r}^{\prime}
$$

where $\overleftrightarrow{\boldsymbol{\chi}}\left(\mathbf{r}, \mathbf{r}^{\prime}, \omega\right)$ is the nonlocal susceptibility tensor. In reasonable good quality QW's the amplitude of the confinement energy fluctuations is typically one order of magnitude smaller than the exciton binding energy. In this limit, disorder affects significantly only the center of mass motion through an effective two dimensional potential $V(\mathbf{R}),{ }^{12,14}$ $\mathbf{R} \equiv(x, y)$ being the projection of the position vector on the plane of the QW. This effective disorder potential felt by excitons, due to interface roughness, impurity concentration and arising from interface imperfections during the growth and/or patterning processes, tends to localize the center of mass motion of QW excitons. 
In QW's described within the usual envelope-function formalism with isotropic electron and hole dispersions and neglecting fine structure splittings, the susceptibility tensor becomes diagonal with identical elements given by $\mu_{v c}^{2}\left|\phi_{1 s}(0)\right|^{2} \rho(z) \rho\left(z^{\prime}\right) G\left(\mathbf{R}, \mathbf{R}^{\prime}, \omega\right)$, where $\mu_{v c}$ is the interband bulk dipole moment, $\phi_{1 s}$ describes the relative electron-hole motion (assumed undistorted by disorder), $\rho(z)$ is the product of the electron and hole confinement functions along the growth axis, and, finally, the quantity $G\left(\mathbf{R}, \mathbf{R}^{\prime}, \omega\right)$ is the retarded propagator for the exciton c.m. motion. The c.m. propagator obeys the following equation:

$$
\left(\hbar \omega+i \delta-\hat{H}_{\mathbf{R}}\right) G\left(\mathbf{R}, \mathbf{R}^{\prime}, \omega\right)=\delta\left(\mathbf{R}-\mathbf{R}^{\prime}\right),
$$

where the effective c.m. Hamiltonian $\hat{H}$, can be written as

$$
\hat{H}_{\mathbf{R}}=-\frac{\hbar^{2} \nabla^{2}}{2 M}+\hbar \omega_{1 s}+V(\mathbf{R})
$$

where $M=m_{e}^{*}+m_{h}^{*}$ is the exciton kinetic mass $\left(m_{e}^{*}\right.$ and $m_{h}^{*}$ are the effective masses of the electron and of the hole) and $\hbar \omega_{1 s}$ is the $1 s$ exciton energy level in the ideal disorder free quantum well. The effective disorder potential $V(\mathbf{R})$ can be modeled as a zero mean, Gauss distributed and spatially correlated process ${ }^{15}$ defined by the property

$$
\left\langle V(\mathbf{R}) V\left(\mathbf{R}^{\prime}\right)\right\rangle=v_{0}^{2} \exp \left(-\left|\mathbf{R}-\mathbf{R}^{\prime}\right|^{2} / 2 \xi^{2}\right),
$$

where $\langle\cdots\rangle$ denotes the ensamble average over random configurations; $v_{0}$ is the width of the energy distribution and $\xi$ is the correlation length characterizing the potential fluctuations. Realistic random potentials can be different from this model, which is however widely adopted because it retains the main physical properties in a very simple way. Inserting the abovementioned expression of $\overleftrightarrow{\boldsymbol{\chi}}$ into Eq. (2), and inserting Eq. (2) into Eq. (1), the total absorbed power in a semiconductor QW can be evaluated according to

$$
\begin{aligned}
\alpha(\omega)= & \mu_{v c}^{2}\left|\phi_{1 s}(0)\right|^{2} \operatorname{Im} \int \widetilde{E}(\mathbf{R}, \omega) G\left(\mathbf{R}, \mathbf{R}^{\prime}, \omega\right) \\
& \times \widetilde{E}\left(\mathbf{R}^{\prime}, \omega\right) d \mathbf{R} d \mathbf{R}^{\prime},
\end{aligned}
$$

where $\widetilde{E}(\mathbf{R}, \omega)=\int \mathbf{E}(\mathbf{r}, \omega) \rho(z) d z$ [we recall that $\left.\mathbf{r} \equiv(\mathbf{R}, z)\right]$. Considering a light field with a given profile centered around the beam position $\mathbf{R}_{0}, \widetilde{E}(\mathbf{R}, \omega)=E_{0}(\omega) g\left(\mathbf{R}-\mathbf{R}_{0}\right)$, we may define a local absorption that is a function of the beam position, and relates the total absorbed power to the power of a local excitation (illumination mode):

$$
\alpha_{g}\left(\mathbf{R}_{0}, \omega\right) \propto \int G^{I}\left(\mathbf{R}, \mathbf{R}^{\prime}, \omega\right) g\left(\mathbf{R}-\mathbf{R}_{0}\right) g\left(\mathbf{R}^{\prime}-\mathbf{R}_{0}\right) d \mathbf{R} d \mathbf{R}^{\prime},
$$

where $G^{I}$ is the imaginary part of $G$. In the following we describe the eventually confined light beam by a Gaussian EM profile $g(\mathbf{R})=\exp \left[-\left(x^{2}+y^{2}\right) / 2 \sigma^{2}\right]$.

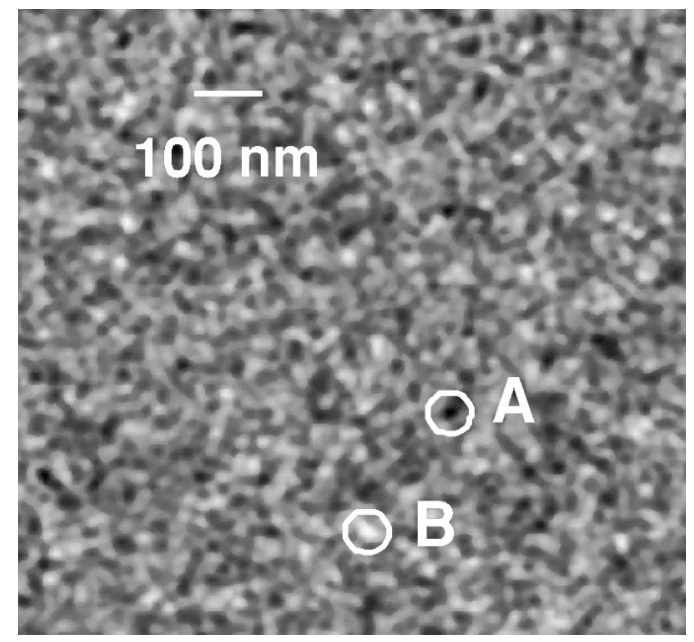

FIG. 1. Specific realization of the disorder potential. The labels A and B specify the locations chosen to calculate the local spectra shown in Fig. 2(a).

\section{NUMERICAL RESULTS}

We perform specific calculations for the total absorption under sample illumination, considering both global $(\sigma=\infty)$ and local absorption spectra for different spatial resolutions. Calculations are carried out in real space, mapping on a fine mesh of points the Hamiltonian (4), which is then tridiagonalized by using the Lanczos algorithm starting from the initial transformation vector $g(\mathbf{r}) .{ }^{8,9}$ Each iteration step produces a new basis state. The iteration in extremely fast due to the sparseness of the Hamiltonian matrix. We truncate the iteration after the spectrum $\alpha_{g}\left(\mathbf{R}_{0}, \omega\right)$ stabilizes. The relevant spectrum is obtained by inversion of the resolvent matrix $(\hbar \omega+i \delta-\hat{H})^{-1}$, which is simple in the tridiagonal form of the Hamiltonian $\hat{H}$. We adopt an exciton kinetic mass of $m=0.25 m_{0}$ typical for GaAs/AlGaAs quantum wells. The spectra have been calculated by considering a square region of $1 \mu \mathrm{m}^{2}$ which has been divided into $n=300 \times 300$ steps; periodic boundary conditions have been adopted. For all the calculated spectra we used a homogeneous broadening fixed at $\delta=30 \mu \mathrm{eV}$, which could be interpreted as the resolution limit of the spectrometer.

Figure 1 displays the specific potential realization of the sample, which has been obtained by using a correlation length $\xi=10 \mathrm{~nm}$ and a value $v_{0}=1.2 \mathrm{meV}$. Figure $2(\mathrm{a})$ shows two local spectra calculated with the beam position centered at two different locations (A and B indicated in Fig. 1 by white circles). In the first case we can note sharp distinct peaks originating from the lowest exciton c.m. states localized inside the local minimum A of the disordered potential, whereas the second spectrum (in proximity of the local maximum) displays a quasicontinuous absorption band arising from higher energy states. These two spectra strongly differ each other and clearly show the capability of near-field spectroscopy in providing direct information on the spatial and energy distribution of excitonic quantum states. ${ }^{8,9}$ In Fig. 2(b) we also report as reference the absorption spectrum obtained under illumination of a larger region of the sample 

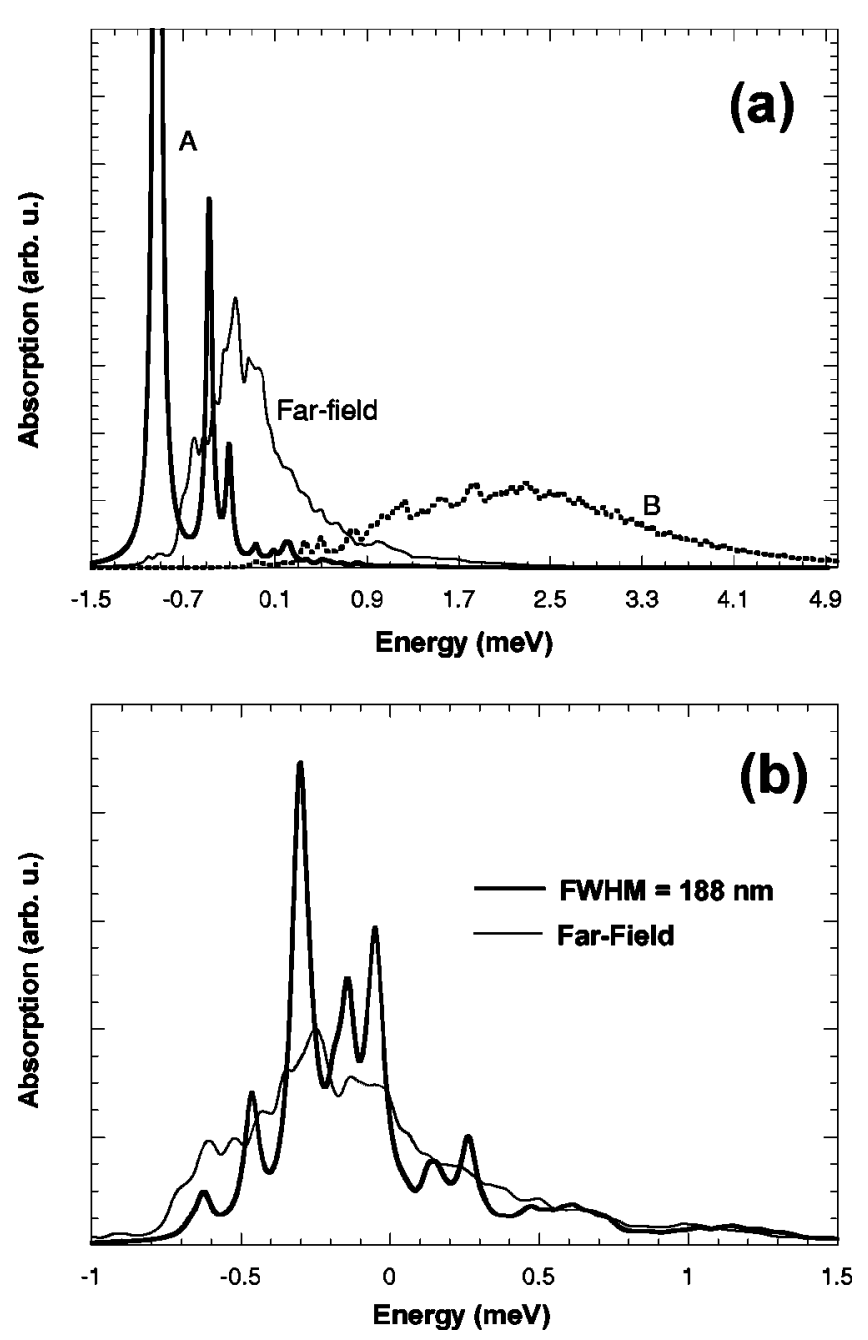

FIG. 2. (a) Far-field absorption spectrum and local absorption spectra $(\mathrm{FWHM}=24 \mathrm{~nm})$ calculated with the beam position centered at the two different locations A and B indicated in Fig. 1 by white circles. (b) Far-field absorption spectrum and local absorption spectrum $(\mathrm{FWHM}=188 \mathrm{~nm})$ obtained under illumination of a larger region, centering the beam at the center of the sample area.

[full width half maximum $(\mathrm{FWHM})=188 \mathrm{~nm}$ ]. The beam was centered at the center of the sample area (Fig. 1).

In order to isolate spectral changes due to excitation of states not accessible by far-field optical probes, we compare far-field absorption spectra (we use $\sigma=\infty$ ) with spatially averaged absorption spectra (Fig. 3). These averaged spectra are composed summing up local spectra obtained centering the confined illuminating beams on a fine mesh of points (with the nearest distance $\Delta_{m} \ll \sigma$ ) on the quantum well plane. Some of the results on GaAs quantum wells are shown in the figure. The obtained global spectrum displays the asymmetric gaussian shape typical of inhomogeneously broadened exciton lines. ${ }^{16}$ The asymmetric shape origins from $k$ vector mixing induced by disorder. We observe that at low spatial resolution (diffraction-limit) averaged spectra coincide almost perfectly with the far-field spectrum. Increasing the spatial resolution, we find significant spectral changes as blueshift and spectral broadening. Thus we find that at subwavelength resolution, summing up local optical spectra

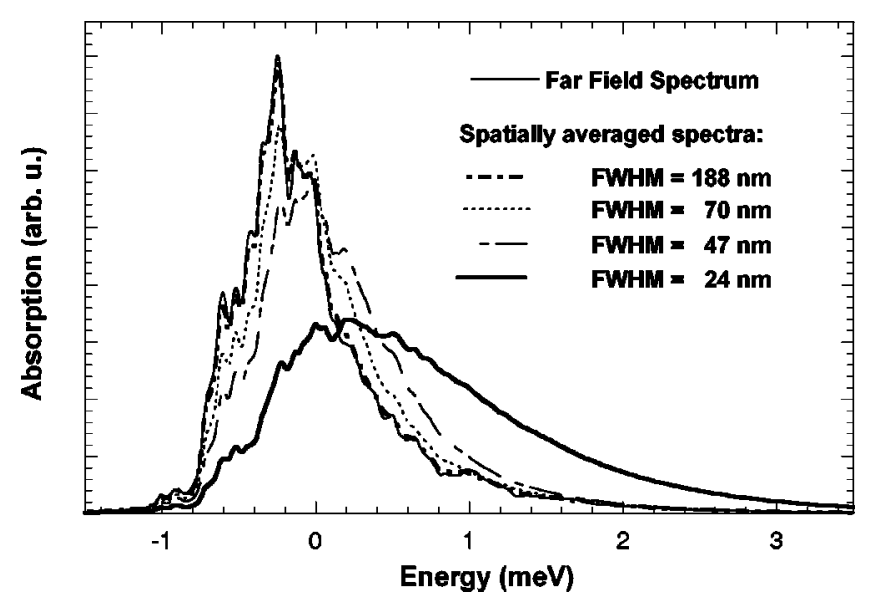

FIG. 3. Far-field absorption spectrum and averaged near-field absorption spectra obtained with different spatial resolutions.

does not reproduce the global spectrum. Although the total excitonic in-plane momentum $K$ is no longer a good quantum number due to disorder, these spectral changes can be understood as a consequence of excitation of high $K$ exciton states not accessible by far-field illumination. We observe that the spectral shape of the low energy region of the spectrum (arising from more localized quantum states) is better maintained at increasing spatial resolutions as compared to the high energy tail.

These results demonstrate that near-field spectroscopy of semiconductor quantum structures does not provide just a spatial selection of inhomogeneous surface systems, in addition it enables the optical detection of states not accessible by far-field optical probes. This ability might be exploited in future experiments on semiconductor quantum structures. It is important to observe that these spectral changes are found at spatially resolutions largely reached by current near-field technology. Recent measurements based on spatially resolved photoluminescence provided direct information on the spatial and energy distribution of light emitting nanometric centers in narrow QW's. ${ }^{1}$ In Fig. 2 they report ${ }^{1}$ the obtained far-field spectrum and the spatially averaged near-field spectrum. The figure clearly shows that the two spectra are far from coinciding and the averaged spectrum presents a pronounced asymmetric high energy tail in contrast to the far-field spectrum and in agreement with the results here presented. However we have to point out that, without accurate checks, it is hard to exclude that these spectral differences do not origin from a different spectral response function of the near- and far-field setup.

It is also interesting to observe that these spectral changes are a direct manifestation of spatial nonlocality [see Eq. (2)]. If we replace the susceptibility function in Eq. (7) with a local susceptibility, $G\left(\mathbf{r}, \mathbf{r}^{\prime}\right) \rightarrow \delta\left(\mathbf{r}-\mathbf{r}^{\prime}\right) G(\mathbf{r})$, it is easy to see from the resulting structure of Eq. (7) that averaged spectra would not differ from far-field spectra. Figure 4 displays numerical results obtained by using the same random potential realization used for all the previous calculations but choosing a larger amplitude $\left(v_{0}=3 \mathrm{meV}\right)$. Comparing Figs. 3 and 4, it emerges that increasing the disorder amplitude produces a lowering of the discrepansies between far-field 


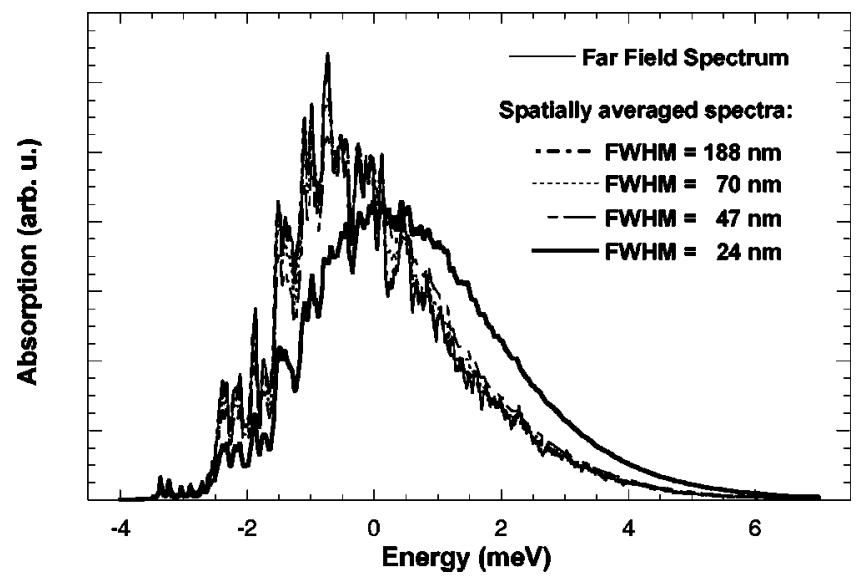

FIG. 4. Far-field absorption spectrum and averaged near-field absorption spectra obtained with the same spatial resolutions of Fig. 3 but choosing a larger amplitude of the random potential $\left(v_{0}\right.$ $=3 \mathrm{meV}$ ).

and spatially averaged spectra. In particular we observe that the averaged spectrum obtained with $\mathrm{FWHM}=47 \mathrm{~nm}$ almost coincides with the far-field spectrum. In contrast the corresponding averaged spectrum in Fig. 3 differs significantly from the far-field spectrum. This result can be interpreted by observing that, increasing disorder, the exciton states suffer a large degree of localization and the system tends to behave as a system with a local susceptibility.

\section{CONCLUSIONS}

The numerical calculations displayed in Fig. 3 show that summing up optical spectra with subwavelength spatial resolutions does not reproduce the global spectrum. This demonstrates that spectral changes at increasing spatial resolutions (due to surface states with high $k$ vectors not accessible by far-field optical excitations) are detectable also in real systems affected by disorder and imperfections. Moreover the spectral changes here described provide a measure of the spatial nonlocality displayed by two-dimensional quantum structures affected by interface fluctuations. Near-field microscopy/spectroscopy of semiconductor quantum structures (due to the nonlocal character of light-matter interaction in these systems) does not provide just a spatial selection of inhomogeneous surface systems, in addition it enables the optical detection of states not accessible by farfield optical probes. This ability could be exploited in future experiments on semiconductor quantum structures. On the contrary diffraction-limited resolutions do not suffice to observe these spectral changes. These results also show that care has to be paid when analyzing line shapes of near-field spectra.

\section{ACKNOWLEDGMENTS}

We are grateful to G. Martino for many useful discussions and suggestions.
${ }^{1}$ H. F. Hess, E. Betzig, T. D. Harris, L. N. Pfeiffer, and K. W. West, Science 264, 1740 (1994).

${ }^{2}$ D. Gammon, E. S. Snow, B. V. Shanabrook, D. S. Katzer, and D. Park, Phys. Rev. Lett. 76, 3005 (1996).

${ }^{3}$ D. Gammon, E. S. Snow, B. V. Shanabrook, D. S. Katzer, and D. Park, Science 273, 87 (1996).

${ }^{4}$ J. Hasen, L. N. Pfeiffer, A. Pinczuk, S. He, K. W. West, and B. S. Dennis, Nature (London) 390, 54 (1997).

${ }^{5}$ J. R. Guest, T. H. Stievater, Gang Chen, E. A. Tabak, B. G. Orr, D. G. Steel, D. Gammon, and D. S. Katzer, Science 293, 2224 (2001).

${ }^{6}$ S. Savasta, G. Martino, and R. Girlanda, Phys. Rev. B 61, 13852 (2000).

${ }^{7}$ A. V. Shchegrov, K. Joulain, R. Carminati, and J. J. Greffet, Phys. Rev. Lett. 85, 1548 (2000).

${ }^{8}$ O. Di Stefano, S. Savasta, G. Martino, and R. Girlanda, Appl.
Phys. Lett. 77, 2804 (2000).

${ }^{9}$ O. Di Stefano, S. Savasta, G. Martino, and R. Girlanda, Phys. Rev. B 62, 11071 (2000).

${ }^{10}$ E. Runge and R. Zimmermann, Phys. Status Solidi B 206, 167 (1998).

${ }^{11}$ C. Weisbuch, R. Dingle, A.C. Grossard, and W. Wiegmann, Solid State Commun. 206, 219 (1981).

${ }^{12}$ R. Zimmermann, Phys. Status Solidi B 173, 129 (1992).

${ }^{13}$ O. Mauritz, G. Goldoni, F. Rossi, and E. Molinari, Phys. Rev. Lett. 82, 847 (1999).

${ }^{14}$ S.D. Baranovskii, U. Doerr, P. Thomas, A. Naumov, and W. Gebhardt, Phys. Rev. B 48, 17149 (1993).

${ }^{15}$ S. Glutsch, D.S. Chemla, and F. Bechstedt, Phys. Rev. B 54, 11 592 (1996).

${ }^{16}$ R.F. Schnabel, R. Zimmermann, D. Bimberg, H. Nickel, R. Lösch, and W. Schlapp, Phys. Rev. B 46, 9873 (1992). 